Agnieszka RYgiel (Kraków)

ŁukAsz StetTneR (Warszawa)

\title{
REMARKS ON SIMPLE ARBITRAGE ON MARKETS WITH BID AND ASK PRICES
}

Abstract. We consider various kinds of simple investment strategies on markets with bid and ask prices. We formulate necessary and sufficient conditions for the absence of arbitrage using those strategies. In the last part of the paper we study the absence of arbitrage for simple strategies without shortselling.

1. Introduction. Let $\left(\Omega, \mathcal{F}, \mathbb{P}, \mathbb{F}=\left(\mathcal{F}_{t}\right)_{t \in[0, T]}\right)$ be a filtered probability space satisfying the usual conditions, i.e. the filtration $\left(\mathcal{F}_{t}\right)_{t \in[0, T]}$ is right continuous and $\mathcal{F}_{0}$ contains all the $\mathbb{P}$-null sets of $\mathcal{F}$. Assume that there exist two $\mathbb{R}^{d}$-valued adapted positive stochastic processes $\underline{S}=\left(\underline{S}_{t}\right)_{t \in[0, T]}$ and $\bar{S}=$ $\left(\bar{S}_{t}\right)_{t \in[0, T]}$, with $\underline{S}_{t}(\omega) \leq \bar{S}_{t}(\omega)$ for all $t \in[0, T]$ and $\omega \in \Omega$, which model the bid (selling) and ask (buying) prices of risky assets. We buy or sell risky assets during the time interval $[0, T]$ liquidating our position just after maturity time $T$ at the instant which we shall denote by $T^{+}$, using the prices $\underline{S}_{T}$ and $\bar{S}_{T}$ respectively. We consider the following different classes of trading strategies.

Definition 1.1. We call an $\mathbb{R}^{d}$-valued process $\Theta=\left(\Theta_{t}\right)_{t \in\left[0, T^{+}\right]}$a simple investment strategy if there exists a positive integer $n \geq 2$ and a finite sequence $\left(\tau_{i}, \theta_{i}\right)_{i=1}^{n}$ of $\mathbb{F}$-stopping times $\tau_{i}$ such that $0=\tau_{1} \leq \cdots \leq \tau_{n}=T$ and of $\mathcal{F}_{\tau_{i}}$-measurable random vectors $\theta_{i} \in \mathbb{R}^{d}$ for $i=1, \ldots, n-1$ with $\theta_{n}=0$ such that $\Theta_{T^{+}}=\theta_{n}=0$ and

$$
\Theta_{t}=\sum_{i=1}^{n-1} \theta_{i} \chi_{\left(\tau_{i}, \tau_{i+1}\right]}(t) .
$$

2010 Mathematics Subject Classification: Primary 91G10; Secondary 60G99, 91B26. Key words and phrases: simple strategies, absence of arbitrage, bid and ask prices. Received 27 June 2016; revised 6 November 2016.

Published online 19 January 2017. 
A simple investment strategy consists of an at most deterministic number of transactions. We shall denote this class of strategies by $\mathcal{S}_{T}$. Note that our simple strategy starts with initial position $\Theta_{0}=0$. We could also consider an initial position $\theta_{0}$ by adding to the right hand side of (1.1) the term $\theta_{0} \chi_{\{0\}}(t)$.

Strategies of the next class consist of a finite but not necessarily deterministically bounded number of transactions.

Definition 1.2. We call an $\mathbb{R}^{d}$-valued process $\Theta=\left(\Theta_{t}\right)_{t \in\left[0, T^{+}\right]}$an almost simple investment strategy if there exists a sequence $\left(\tau_{i}, \theta_{i}\right)$ of $\mathbb{F}$ stopping times $0=\tau_{1} \leq \cdots \leq \tau_{s}=T$, where $s=\inf \left\{i \geq 1: \tau_{i}=T\right\}$ and $\mathbb{P}(s<\infty)=1$, and of $\mathcal{F}_{\tau_{i}}$-measurable random vectors $\theta_{i} \in \mathbb{R}^{d}$ for $i \geq 1$ with $\theta_{s}=0$ such that $\Theta_{T^{+}}=\theta_{s}=0$ and

$$
\Theta_{t}=\sum_{i=1}^{\infty} \theta_{i} \chi_{\left(\tau_{i}, \tau_{i+1}\right]}(t) .
$$

The class of almost simple strategies will be denoted by $a \mathcal{S}_{T}$. The above two classes pretty well approximate reasonable investment strategies in the case of continuous bid and ask prices (e.g. functions of fraction Brownian motion, see [4]). In the case of càdlàg bid and ask prices we may have additional gain when jumps of the bid and ask prices are predictable, and therefore following [5] we have to consider left and right transactions: just before the jump and after the jump.

Definition 1.3. We call an $\mathbb{R}^{d}$-valued process $\Theta=\left(\Theta_{t}\right)_{t \in\left[0, T^{+}\right]}$a generalized simple investment strategy if there exists a positive integer $n \geq 2$ and a finite sequence $\left(\tau_{i}, \theta_{i}{ }^{-}, \theta_{i}\right)_{i=1}^{n}$ of $\mathbb{F}$-stopping times $\tau_{i}$ such that $0=\tau_{1} \leq$ $\cdots \leq \tau_{n}=T$ and of $\mathcal{F}_{\tau_{i}^{-}}$-measurable random vectors $\theta_{i}^{-}$and $\mathcal{F}_{\tau_{i}}$-measurable random vectors $\theta_{i} \in \mathbb{R}^{d}$ for $i=1, \ldots, n-1$ with $\theta_{1}{ }^{-}=\theta_{n}=0$ such that $\Theta_{T^{+}}=\theta_{n}=0$ and

$$
\Theta_{t}=\sum_{i=2}^{n} \theta_{i}^{-} \chi_{\left\{\tau_{i}\right\}}(t)+\sum_{i=1}^{n-1} \theta_{i} \chi_{\left(\tau_{i}, \tau_{i+1}\right)}(t),
$$

and we have $\theta_{i}^{-}=\theta_{i-1}$ on the totally inaccessible part of $\tau_{i}$ for $i=2, \ldots, n$.

The class of such strategies will be denoted by $g \mathcal{S}_{T}$. Each strategy in this class consists of a deterministically finite number of left and right transactions.

The next class will be a natural extension of the class with a finite number of transactions.

Definition 1.4. We call an $\mathbb{R}^{d}$-valued process $\Theta=\left(\Theta_{t}\right)_{t \in\left[0, T^{+}\right]}$a generalized almost simple investment strategy if there exists a positive integer $n \geq 2$ and a sequence $\left(\tau_{i}, \theta_{i}{ }^{-}, \theta_{i}\right)$ of $\mathbb{F}$-stopping times $\tau_{i}$ such that $0=\tau_{1} \leq \cdots \leq \tau_{s}=T$, where $s=\inf \left\{i \geq 1: \tau_{i}=T\right\}$ and $\mathbb{P}(s<\infty)=1$, and 
of $\mathcal{F}_{\tau_{i}^{-}}$-measurable random vectors $\theta_{i}{ }^{-}$and $\mathcal{F}_{\tau_{i}}$-measurable random vectors $\theta_{i} \in \mathbb{R}^{d}$ for $i \geq 1$ with $\theta_{1}{ }^{-}=\theta_{s}=0$ such that $\Theta_{T^{+}}=\theta_{s}=0$ and

$$
\Theta_{t}=\sum_{i=2}^{\infty} \theta_{i}^{-} \chi_{\left\{\tau_{i}\right\}}(t)+\sum_{i=1}^{\infty} \theta_{i} \chi_{\left(\tau_{i}, \tau_{i+1}\right)}(t),
$$

and we have $\theta_{i}^{-}=\theta_{i-1}$ on the totally inaccessible part of $\tau_{i}$ for $i \geq 2$.

The class of such strategies will be denoted by $\mathrm{gaS}_{T}$. As in the case of almost simple strategies, each generalized almost simple strategy consists of a finite number of transactions, but this number may depend on $\omega \in \Omega$.

REMARK 1.5. In [5] generalized almost simple strategies were required to be predictable processes. In that case by [9, Theorem 2.24 and its proof] the process $\Theta$ does not have jumps at totally inaccessible stopping times. Consequently, $\theta_{i}^{-}=\theta_{i-1}$ on the totally inaccessible part of $\tau_{i}$ for $i=2, \ldots, s$. This is intuitively clear since we are not able to predict totally inaccessible stopping times to make profit at those times by an immediate portfolio change.

Definition 1.6. The wealth process $V(\Theta)=\left(V_{t}(\Theta)\right)_{t \in[0, T]}$ generated by the trading strategy $\Theta \in g a \mathcal{S}_{T}$ of the form (1.4) is given by

$$
\begin{aligned}
V_{t}(\Theta)= & \sum_{i=2}^{\infty} \chi_{\left\{\tau_{i} \leq t\right\}}\left[-\left(\theta_{i}^{-}-\theta_{i-1}\right)^{+} \bar{S}_{\tau_{i}^{-}}+\left(\theta_{i}^{-}-\theta_{i-1}\right)^{-} \underline{S}_{\tau_{i}^{-}}\right] \\
& +\sum_{i=1}^{\infty} \chi_{\left\{\tau_{i}<t\right\}}\left[-\left(\theta_{i}-\theta_{i}^{-}\right)^{+} \bar{S}_{\tau_{i}}+\left(\theta_{i}-\theta_{i}^{-}\right)^{-} \underline{S}_{\tau_{i}}\right] \\
& +\Theta_{t}^{+} \underline{S}_{t}-\Theta_{t}^{-} \bar{S}_{t} .
\end{aligned}
$$

Notice that the right hand side of the first line in 1 1.5) corresponds to the gain or cost of changing the strategy just before time $\tau_{i}$ (left transaction) from $\theta_{i-1}$ to $\theta_{i}^{-}$. In the second line of 1.5 we have the gain or cost associated with the change of portfolio just after time $\tau_{i}$ (right transaction). The third line corresponds to liquidation of our financial position at time $t$. We only allow left transaction at time $t$. Notice furthermore that for $s=\inf \left\{i \geq 1: \tau_{i}=T\right\}$, assuming that $\theta_{s}=0$ we have

$$
\begin{aligned}
V_{T}(\Theta)= & \sum_{i=2}^{s}\left[-\left(\theta_{i}^{-}-\theta_{i-1}\right)^{+} \bar{S}_{\tau_{i}^{-}}+\left(\theta_{i}^{-}-\theta_{i-1}\right)^{-} \underline{S}_{\tau_{i}^{-}}\right] \\
& +\sum_{i=1}^{s}\left[-\left(\theta_{i}-\theta_{i}^{-}\right)^{+} \bar{S}_{\tau_{i}}+\left(\theta_{i}-\theta_{i}^{-}\right)^{-} \underline{S}_{\tau_{i}}\right] .
\end{aligned}
$$


In particular, if $\Theta \in a \mathcal{S}_{T}$, then

$$
V_{T}(\Theta)=\sum_{i=1}^{s}\left[-\left(\theta_{i}-\theta_{i-1}\right)^{+} \bar{S}_{\tau_{i}}+\left(\theta_{i}-\theta_{i-1}\right)^{-} \underline{S}_{\tau_{i}}\right]
$$

where $\theta_{0}=0$. Furthermore we have

REMARK 1.7. It is clear that $\mathcal{S}_{T} \subset a \mathcal{S}_{T}, \mathcal{S}_{T} \subset g \mathcal{S}_{T}$ and $a \mathcal{S}_{T} \subset g a \mathcal{S}_{T}$.

The following example explains the meaning of generalized simple strategies.

EXAMPLE 1.8. Assume we are given a fractional Brownian motion $\left(B_{t}^{H}\right)$ (or any continuous stochastic process) and three independent random variables: $Z$ taking values in $\{0,1\}$ with $\mathbb{P}\{Z=0\} \in(0,1), \xi$, and an exponentially distributed random variable $\eta$ such that $\mathbb{P}\{\eta \geq t\}=e^{1-t}$ for $t \geq 1$. Let $\underline{S}_{t}=(1-\mu) S_{t}$ and $\bar{S}_{t}=(1+\lambda) S_{t}$ for $\mu, \lambda>0$ and $t \geq 0$, where $S_{t}=e^{B_{t}^{H}}$ for $t \in[0,1]$. If $Z=0$ we define $\left(S_{t}\right)$ for $t \geq 1$ as follows: let

$$
\sigma:=\inf \left\{t \geq 1:\left|B_{t}^{H}-B_{1}^{H}\right| \geq 1\right\}
$$

and for $t<\sigma$ set $S_{t}=e^{B_{t}^{H}}$, while for $t \geq \sigma$ define $S_{t}=e^{B_{t}^{H}+\xi}$. If $Z=1$ we let $S_{t}=e^{B_{t}^{H}}$ for $t<\eta$ and $S_{t}=e^{B_{t}^{H}+\xi}$ for $t \geq \eta$. Assume now that the $i$ th transaction (for an $i \geq 2$ ) is at time $\tau_{i}$ where $\tau_{i}=\sigma$ on $\{Z=0\}$ and $\tau_{i}=\eta$ on $\{Z=1\}$. Then the processes $\left(\underline{S}_{t}\right)$ and $\left(\bar{S}_{t}\right)$ are càdlàg with only one jump at $\tau_{i}$. The stopping time $\tau_{i}^{\{Z=0\}}$ equal to $\tau_{i}$ on $\{Z=0\}$ and $\infty$ otherwise is an accessible part of $\tau_{i}$ (in fact even predictable). The stopping time $\tau_{i}^{\{Z=1\}}$ (defined as above) is a totally inaccessible part of $\tau_{i}$. Left transaction at time $\tau_{i}$ makes sense on the event $\{Z=0\}$ since the corresponding part of the wealth process

$$
-\left(\theta_{i}^{-}-\theta_{i-1}\right)^{+} \bar{S}_{\tau_{i}^{-}}+\left(\theta_{i}^{-}-\theta_{i-1}\right)^{-} \underline{S}_{\tau_{i}^{-}}
$$

is a limit of the wealth processes $-\left(\theta_{i}^{-}-\theta_{i-1}\right)^{+} \bar{S}_{\tau_{i}^{n}}+\left(\theta_{i}^{-}-\theta_{i-1}\right)^{-} \underline{S}_{\tau_{i}^{n}}$, where $\tau_{1}^{n}=\inf \left\{t \geq 1:\left|B_{t}^{H}-B_{1}^{H}\right| \geq 1-n^{-1}\right\}$, assuming that $\theta_{i}^{-}$are $\mathcal{F}_{\tau_{i}^{n}}$-measurable. We are not able to approximate the wealth process on the event $\{Z=1\}$ in a similar way since we cannot approximate $\eta$ from below using stopping times.

For continuous processes we can restrict ourselves to the classes $\mathcal{S}_{T}$ and $a \mathcal{S}_{T}$. Namely we have

LEMma 1.9. If the bid and ask prices are continuous processes then it is not optimal to have $\theta_{i}^{-}$different from $\theta_{i-1}$, so that we should have only right transactions and consequently the classes $\mathcal{S}_{T}$ and $a \mathcal{S}_{T}$ are sufficient to study arbitrage. 
Proof. If the bid and ask prices are continuous then we have $\underline{S}_{\tau_{i}}^{-}=\underline{S}_{\tau_{i}}$ and $\bar{S}_{\tau_{i}}^{-}=\bar{S}_{\tau_{i}}$, from which in 1.5 we obtain for $\tau_{i}<t$ the sum

$$
\begin{aligned}
- & {\left[\left(\theta_{i}^{-}-\theta_{i-1}\right)^{+}+\left(\theta_{i}-\theta_{i}^{-}\right)^{+}\right] \bar{S}_{\tau_{i}} } \\
& +\left[\left(\theta_{i}^{-}-\theta_{i-1}\right)^{-}+\left(\theta_{i}-\theta_{i}^{-}\right)^{-}\right] \underline{S}_{\tau_{i}} \\
= & -\left(\theta_{i}-\theta_{i-1}\right) \bar{S}_{\tau_{i}}-\left[\left(\theta_{i}^{-}-\theta_{i-1}\right)^{-}+\left(\theta_{i}-\theta_{i}^{-}\right)^{-}\right]\left(\bar{S}_{\tau_{i}}-\underline{S}_{\tau_{i}}\right) \\
\leq & -\left(\theta_{i}-\theta_{i-1}\right) \bar{S}_{\tau_{i}}-\left(\theta_{i}-\theta_{i-1}\right)^{-}\left(\bar{S}_{\tau_{i}}-\underline{S}_{\tau_{i}}\right) \\
= & -\left(\theta_{i}-\theta_{i-1}\right)^{+} \bar{S}_{\tau_{i}}+\left(\theta_{i}-\theta_{i-1}\right)^{-} \underline{S}_{\tau_{i}},
\end{aligned}
$$

and inequality can be replaced by equality whenever either $\theta_{i-1} \geq \theta_{i}^{-} \geq \theta_{i}$ or $\theta_{i} \geq \theta_{i}^{-} \geq \theta_{i-1}$. In both cases we can directly change the portfolio from $\theta_{i-1}$ to $\theta_{i}$, so that left transactions are not necessary. When $\tau_{i}=t$ there is no reason to change the portfolio since we immediately liquidate it.

Definition 1.10. A strategy $\Theta \in g a \mathcal{S}_{T}$ is

(i) an arbitrage if

$$
V_{T}(\Theta) \geq 0 \quad \mathbb{P} \text {-a.s. } \quad \text { and } \quad \mathbb{P}\left(V_{T}(\Theta)>0\right)>0 ;
$$

(ii) an obvious arbitrage if there exist stopping times $\sigma$ and $\tau$ and an $\mathcal{F}_{\sigma}$-measurable random vector $\theta \in \mathbb{R}^{d}$ such that $\Theta$ is given by

$$
\Theta_{t}=\theta \chi_{(\sigma, \tau]}(t), \quad t \in[0, T],
$$

and satisfies 1.9.

Definition 1.11. We say that a market satisfies

(i) the absence of simple arbitrage (ASA) if

$$
\Theta \in \mathcal{S}_{T} \text { and } \mathbb{P}\left(V_{T}(\Theta) \geq 0\right)=1 \Rightarrow V_{T}(\Theta)=0 \mathbb{P} \text {-a.s.; }
$$

(ii) the absence of almost simple arbitrage (AaSA) if

$$
\Theta \in a \mathcal{S}_{T} \text { and } \mathbb{P}\left(V_{T}(\Theta) \geq 0\right)=1 \Rightarrow V_{T}(\Theta)=0 \mathbb{P} \text {-a.s.; }
$$

(iii) the absence of generalized simple arbitrage (AgSA) if

$$
\Theta \in g \mathcal{S}_{T} \text { and } \mathbb{P}\left(V_{T}(\Theta) \geq 0\right)=1 \Rightarrow V_{T}(\Theta)=0 \mathbb{P} \text {-a.s.; }
$$

(iv) the absence of generalized almost simple arbitrage (AgaSA) if

$$
\Theta \in \text { gaS }_{T} \text { and } \mathbb{P}\left(V_{T}(\Theta) \geq 0\right)=1 \Rightarrow V_{T}(\Theta)=0 \mathbb{P} \text {-a.s.; }
$$

(v) the absence of obvious arbitrage (AOA) if for any stopping times $\sigma$ and $\tau$ and every $\mathcal{F}_{\sigma}$-measurable random vector $\theta \in \mathbb{R}^{d}$,

$$
\Theta=\theta \chi_{(\sigma, \tau]} \text { and } \mathbb{P}\left(V_{T}(\Theta) \geq 0\right)=1 \Rightarrow V_{T}(\Theta)=0 \mathbb{P} \text {-a.s.; }
$$

REMARK 1.12. It is clear that AgaSA $\Rightarrow \mathrm{AaSA} \Rightarrow \mathrm{ASA}$ and $\mathrm{AgaSA} \Rightarrow \mathrm{AgSA}$ $\Rightarrow$ ASA. 
In this paper we are interested in problems of arbitrage for general continuous time càdlàg processes. As is shown in [14], the fundamental notion of no free lunch with vanishing risk in Delbaen-Schachermayer arbitrage theory restricts price processes to the class of semimartingales. Since a number of financial market models (in particular in the model with transaction costs) can be constructed using processes which are not semimartingales, e.g. functions of fractional Brownian motion [3], there is a natural need to study arbitrage without semimartingales, as was pointed out in [4, 10]. Such approaches use simple strategies to characterize arbitrage. Various results concerning the absence of arbitrage for general bid and ask prices which are mainly continuous processes were formulated in [1, 2, 6, 7, 11]. When bid and ask prices are only càdlàg processes, the class of simple strategies has to be enlarged to include the so called left and right transactions, as was pointed out in [5].

This paper generalizes [13] in various directions. First of all, instead of proportional transaction costs we consider bid and ask prices which are general càdlàg. Following [5] we also consider generalized simple strategies. We formulate four kinds of simple strategies allowing even a finite but random number of transactions in a finite time interval $[0, T]$ and study four kinds of arbitrage. We extend conditions introduced in [13] and show examples which prove that a finite but random number of transactions may lead to arbitrage. In particular in Section 2 we introduce conditions (B) and (C) which are necessary and sufficient for the absence of simple arbitrage. In Section 3 we formulate condition (D) which is sufficient for the absence of arbitrage with respect to generalized almost simple strategies.

In the last section we study the absence of arbitrage for simple strategies without shortselling. This is the case where we are not allowed to trade borrowed assets. We give conditions (E) and (F) under which we have the absence of arbitrage for simple strategies without shortselling.

The paper contains various generalizations of formerly proved results. In such cases we indicate the original source before stating its extension.

Note that the absence of simple arbitrage is not equivalent to the absence of arbitrage over almost simple strategies. First we consider a market without transaction costs with infinite time horizon. The following example exhibits a nonnegative martingale that admits an almost simple arbitrage opportunity.

ExAmPle 1.13. Let $y(0)=1$ and $\mathbb{P}(y(n)=1)=1 / 2, \mathbb{P}(y(n)=-1)=$ $1 / 2$, with $(y(n))$ i.i.d. Let

$$
z(t)=\sum_{i=0}^{k} y(i) \quad \text { for } k \leq t<k+1
$$


and

$$
\tau=\inf \{i>0: y(i)=-1\}
$$

Define

$$
X_{t}=z(t \wedge \tau) \quad \text { for } t \in[0, \infty) .
$$

Then $\left(X_{t}\right)_{t \in[0, \infty)}$, as a stopped martingale, is a martingale (note that $z$ is a martingale with right continuous trajectories and $\tau$ is a stopping time). Moreover, $z(\tau)$ is an integrable random variable. Indeed,

$$
\begin{aligned}
\mathbb{E}(z(\tau)) & =\mathbb{E}\left[\sum_{i=1}^{\infty} z(i) \chi_{\{\tau=i\}}\right]=\sum_{i=1}^{\infty} z(i) \mathbb{P}(\tau=i) \\
& =\sum_{i=1}^{\infty}(i-1)\left(\frac{1}{2}\right)^{i}=\sum_{i=1}^{\infty} i\left(\frac{1}{2}\right)^{i+1}=1
\end{aligned}
$$

Let $\underline{S}_{t}=\bar{S}_{t}=X_{t}$. Consider the strategy

$$
\Theta_{t}=\sum_{n=0}^{\infty}\left(-2^{n}\right) \chi_{(n, n+1]}(t) \chi_{\{t<\tau\}}
$$

This strategy vanishes after the stopping time $\tau$, which is finite:

$$
\mathbb{P}(\tau<\infty)=\sum_{i=1}^{\infty} \mathbb{P}(\tau=i)=\sum_{i=1}^{\infty}\left(\frac{1}{2}\right)^{i}=1
$$

Note that the gain on $\{\tau=n+1\}$ is given by

$$
1+\sum_{i=1}^{n}\left(2^{i}-2^{i-1}\right)(i+1)=1+\sum_{i=1}^{n} 2^{i-1}(i+1)=1+n 2^{n},
$$

while the payment is $n 2^{n}\left(2^{n}\right.$ stocks at price $\left.z(\tau) \chi_{\{\tau=n+1\}}=n\right)$, so that the final wealth process of the strategy $\Theta$ is equal to 1 .

Below we adapt the above example to the case of finite time horizon $(t \in[0,1])$.

ExAmPle 1.14. Let $y(0)=1$ and $\mathbb{P}(y(n)=1)=1 / 2, \mathbb{P}(y(n)=-1)=$ $1 / 2$, with $(y(n))$ i.i.d. Let $z(t)=\sum_{i=0}^{k} y(i)$ for $k \leq t<k+1$. Define

$$
S_{t}=z(\tan (t \pi / 2) \wedge \tau) \text { for } t<1
$$

and $S_{1}=z(\tau)$ with $\tau=\inf \{t>0: y([t])=-1\}$. Then $S$ is a martingale and there is no arbitrage over simple strategies (see [13, Lemma 2.15 and Theorem 2.18]). An almost simple arbitrage strategy is given by

$$
\Theta_{t}=\sum_{n=0}^{\infty}\left(-2^{n}\right) \chi_{((2 / \pi) \arctan (n),(2 / \pi) \arctan (n+1)]}(t) \chi_{\{t<\tau\}} .
$$

The following example shows that $(\mathrm{ASA}) \nRightarrow(\mathrm{AaSA})$ on a market with bid and ask prices and infinite-time horizon. 
EXAMPLE 1.15. Let $X$ be a martingale given in Example 1.13. Let $a_{i}=$ $2^{-i-1}$ for $i \geq 0$ and $b_{i}=2^{-2 i}$ for $i \geq 1, b_{0}=1 / 4$. Define

$$
\bar{S}_{t}=X_{t}+\sum_{i=0}^{\infty} a_{i} \chi_{[i, i+1)}(t) \quad \text { and } \quad \underline{S}_{t}=X_{t}-\sum_{i=0}^{\infty} b_{i} \chi_{[i, i+1)}(t) .
$$

An almost simple arbitrage strategy is

$$
\Theta_{t}=\sum_{n=0}^{\infty}\left(-2^{n}\right) \chi_{(n, n+1]}(t) \chi_{\{t<\tau\}} .
$$

Clearly, the gain on $\{\tau=n+1\}$ is given by

$$
1+n 2^{n}-\left[b_{0}+\sum_{i=1}^{n}\left(2^{i}-2^{i-1}\right) b_{i}\right]=n 2^{n}+\frac{1}{4}+2^{-n-1}
$$

and the payment on $\{\tau=n+1\}$ is

$$
n 2^{n}+a_{n+1} 2^{n}=n 2^{n}+1 / 4 .
$$

Consequently, $V_{\infty}(\Theta)=1 / 2^{\tau}>0$ a.s.

We continue our investigation by showing that there is a simple arbitrage if and only if there exists an obvious arbitrage. This result, which is in fact based on Rola's paper [12], does not seem to be commonly known for continuous time markets with transaction costs. In [12, Theorem 2] equivalent conditions for the absence of arbitrage in discrete time markets with bid and ask prices are presented. In particular, the absence of arbitrage on markets with bid and ask prices is equivalent to the absence of arbitrage between two transaction times.

THEOREM 1.16. The following conditions are equivalent:

(i) there is no simple arbitrage;

(ii) there is no obvious arbitrage.

Proof. It suffices to show that for transaction times $\tau_{1}, \ldots, \tau_{n}$ the following conditions are equivalent:

(a) there is no arbitrage for transaction times $0=\tau_{1} \leq \cdots \leq \tau_{n} \leq T$;

(b) there is no arbitrage for any two transaction times $\sigma_{1} \leq \sigma_{2}$ taking values in $\left\{\tau_{1}, \ldots, \tau_{n}\right\}$.

(a) $\Rightarrow(\mathrm{b})$. Trivial.

(b) $\Rightarrow\left(\right.$ a). Assume that there exists a simple arbitrage $\Theta=\left(\Theta_{t}\right)_{t \in[0, T]}$ where $\Theta_{t}=\sum_{i=1}^{n-1} \theta_{i} \chi_{\left(\tau_{i}, \tau_{i+1}\right]}(t)$. Then the discrete time market with price processes $\underline{X}=\left(\underline{X}_{i}\right)_{i=1}^{n}$ and $\bar{X}=\left(\bar{X}_{i}\right)_{i=1}^{n}$ with $\underline{X}_{i}=\underline{S}_{\tau_{i}}$ and $\bar{X}_{i}=\bar{S}_{\tau_{i}}$ for $i=1, \ldots, n$ admits an arbitrage opportunity. By [12, Theorem 2] there exists an arbitrage on a market with bid and ask processes $\left\{\left(\underline{X}_{i}, \bar{X}_{i}\right),\left(\underline{X}_{i+k}, \bar{X}_{i+k}\right)\right\}$ 
for some $1 \leq i \leq i+k \leq T$. Setting $\sigma_{1}=\tau_{i}$ and $\sigma_{2}=\tau_{i+k}$, we have an arbitrage for two transaction times taking values in $\left\{\tau_{1}, \ldots, \tau_{n}\right\}$.

2. Arbitrage for simple strategies on the market with bid and ask prices. In this section we show necessary and sufficient conditions for the absence of arbitrage for simple strategies.

Definition 2.1. We say that $(\underline{S}, \bar{S})$ satisfies condition (B) if for every stopping time $\sigma$ such that $\mathbb{P}(\sigma<T)>0$ and for every $\epsilon>0$ we have

$$
\mathbb{P}\left(\sup _{t \in[\sigma, T]}\left[\theta^{+}\left(\underline{S}_{t}-\bar{S}_{\sigma}\right)-\theta^{-}\left(\bar{S}_{t}-\underline{S}_{\sigma}\right)\right]<\epsilon \mid \mathcal{F}_{\sigma}\right)>0 \quad \mathbb{P} \text {-a.s. }
$$

for every $\mathcal{F}_{\sigma}$-measurable $d$-dimensional random vector $\theta$ such that $|\theta|=1$ $\mathbb{P}$-almost surely, with $|\cdot|$ standing for the Euclidean norm in $\mathbb{R}^{d}$.

The following lemma shows that condition (B) is necessary for the absence of arbitrage over simple strategies on the market with bid and ask prices $(\underline{S}, \bar{S})$.

Lemma 2.2. Let $\underline{S}$ and $\bar{S}$ be adapted càdlàg processes. If there is no obvious arbitrage with bid and ask processes $\underline{S}$ and $\bar{S}$, then $(\underline{S}, \bar{S})$ satisfies condition (B).

A similar result for markets without transaction costs is shown in [1] and [13].

Proof. Assume $\underline{S}$ and $\bar{S}$ do not admit an obvious arbitrage opportunity and $(\underline{S}, \bar{S})$ does not satisfy condition (B). Then there is a stopping time $\sigma$ with $\sigma \leq T$ a.s. and $\epsilon>0$ and an $\mathcal{F}_{\sigma}$-measurable random variable $\theta$ such that $\mathbb{P}\left(\sup _{t \in[\sigma, T]}\left[\theta^{+}\left(\underline{S}_{t}-\bar{S}_{\sigma}\right)-\theta^{-}\left(\bar{S}_{t}-\underline{S}_{\sigma}\right)\right]<\epsilon \mid \mathcal{F}_{\sigma}\right)=0$ with positive probability. Let

$$
A=\left\{\mathbb{P}\left(\sup _{t \in[\sigma, T]}\left[\theta^{+}\left(\underline{S}_{t}-\bar{S}_{\sigma}\right)-\theta^{-}\left(\bar{S}_{t}-\underline{S}_{\sigma}\right)\right]<\epsilon \mid \mathcal{F}_{\sigma}\right)=0\right\} .
$$

Then clearly $A \in \mathcal{F}_{\sigma}$ and $\mathbb{P}(A)>0$. So

$$
\mathbb{P}\left(A \cap\left\{\sup _{t \in[\sigma, T]}\left[\theta^{+}\left(\underline{S}_{t}-\bar{S}_{\sigma}\right)-\theta^{-}\left(\bar{S}_{t}-\underline{S}_{\sigma}\right)\right]<\epsilon\right\}\right)=0
$$

and

(2.2) $\sup _{t \in[\sigma, T]}\left[\theta^{+}\left(\underline{S}_{t}-\bar{S}_{\sigma}\right)-\theta^{-}\left(\bar{S}_{t}-\underline{S}_{\sigma}\right)\right] \geq \epsilon \quad$ on $A$ with probability one.

Define

$$
\tilde{\sigma}= \begin{cases}\sigma & \text { on } A \\ T & \text { on } A^{c}\end{cases}
$$

Since $A \in \mathcal{F}_{\sigma}, \tilde{\sigma}$ is a stopping time. Define

$$
\tau=\inf \left\{t \geq \tilde{\sigma}: \theta^{+}\left(\underline{S}_{t}-\bar{S}_{\tilde{\sigma}}\right)-\theta^{-}\left(\bar{S}_{t}-\underline{S}_{\tilde{\sigma}}\right)>\epsilon / 2\right\} .
$$


As $\underline{S}$ and $\bar{S}$ have right continuous paths, we have $\theta^{+}\left(\underline{S}_{\tau}-\bar{S}_{\tilde{\sigma}}\right)-\theta^{-}\left(\bar{S}_{\tau}-\underline{S}_{\tilde{\sigma}}\right)$ $\geq \epsilon / 2$ on $A$ with probability one. Moreover $\tau \leq T$ almost surely on $A$ : otherwise $\theta^{+}\left(\underline{S}_{t}-\bar{S}_{\tilde{\sigma}}\right)-\theta^{-}\left(\bar{S}_{t}-\underline{S}_{\tilde{\sigma}}\right)<\epsilon / 2$ on $A$ with positive probability for any $t \in[\tilde{\sigma}, T]$, in contradiction with $(2.2)$. This means that $\theta^{+}\left(\underline{S}_{\tau}-\bar{S}_{\tilde{\sigma}}\right)-$ $\theta^{-}\left(\bar{S}_{\tau}-\underline{S}_{\tilde{\sigma}}\right)>0$ on $A$, so $\chi_{A}\left(\theta^{+}\left(\underline{S}_{\tau}-\bar{S}_{\tilde{\sigma}}\right)-\theta^{-}\left(\bar{S}_{\tau}-\underline{S}_{\tilde{\sigma}}\right)\right) \geq 0$ almost surely and $\mathbb{P}\left(\chi_{A}\left(\theta^{+}\left(\underline{S}_{\tilde{\tau}}-\bar{S}_{\tilde{\sigma}}\right)-\theta^{-}\left(\bar{S}_{\tilde{\tau}}-\underline{S}_{\tilde{\sigma}}\right)\right)>0\right)>0$ for

$$
\tilde{\tau}= \begin{cases}\tau & \text { on } A \\ T & \text { on } A^{c}\end{cases}
$$

In other words, the investment strategy $\Theta=\theta \chi_{A} \chi_{(\tilde{\sigma}, \tilde{\tau}]}$ is an obvious arbitrage, which contradicts our assumption.

Definition 2.3. We say that $(\underline{S}, \bar{S})$ satisfies condition (C) if for every stopping time $\sigma$ such that $\mathbb{P}(\sigma<T)>0$ and for every $\epsilon>0$ we have

$$
\mathbb{P}\left(\inf _{t \in[\sigma, T]}\left[\theta^{+}\left(\underline{S}_{t}-\bar{S}_{\sigma}\right)-\theta^{-}\left(\bar{S}_{t}-\underline{S}_{\sigma}\right)\right]>-\epsilon \mid \mathcal{F}_{\sigma}\right)>0 \quad \mathbb{P} \text {-a.s. }
$$

for every $\mathcal{F}_{\sigma}$-measurable random variable $\theta$ such that $|\theta|=1 \mathbb{P}$-almost surely.

REMARK 2.4. $(\underline{S}, \bar{S})$ satisfies condition $(\mathrm{C})$ if and only if for every stopping time $\sigma$ such that $\mathbb{P}(\sigma<T)>0$ and for every $\epsilon>0$ we have

$$
\mathbb{P}\left(\sup _{t \in[\sigma, T]}\left[\theta^{+}\left(\bar{S}_{t}-\underline{S}_{\sigma}\right)-\theta^{-}\left(\underline{S}_{t}-\bar{S}_{\sigma}\right)\right]<\epsilon \mid \mathcal{F}_{\sigma}\right)>0 \quad \mathbb{P} \text {-a.s. }
$$

for every $\mathcal{F}_{\sigma}$-measurable random vector $\theta$ such that $|\theta|=1 \mathbb{P}$-almost surely. Clearly $-\theta$ is also an $\mathcal{F}_{\sigma}$-measurable random variable such that $|\theta|=1$ $\mathbb{P}$-almost surely, and by condition $(\mathrm{C})$ we have

$$
\mathbb{P}\left(\inf _{t \in[\sigma, T]}\left[(-\theta)^{+}\left(\underline{S}_{t}-\bar{S}_{\sigma}\right)-(-\theta)^{-}\left(\bar{S}_{t}-\underline{S}_{\sigma}\right)\right]>-\epsilon \mid \mathcal{F}_{\sigma}\right)>0 \quad \mathbb{P} \text {-a.s. }
$$

Note that $(-\theta)^{+}\left(\underline{S}_{t}-\bar{S}_{\sigma}\right)-(-\theta)^{-}\left(\bar{S}_{t}-\underline{S}_{\sigma}\right)=-\left[\theta^{+}\left(\bar{S}_{t}-\underline{S}_{\sigma}\right)-\theta^{-}\left(\underline{S}_{t}-\bar{S}_{\sigma}\right)\right]$ and

$$
\begin{aligned}
\left\{\operatorname { i n f } _ { t \in [ \sigma , T ] } \left[(-\theta)^{+}\left(\underline{S}_{t}-\bar{S}_{\sigma}\right)\right.\right. & \left.\left.-(-\theta)^{-}\left(\bar{S}_{t}-\underline{S}_{\sigma}\right)\right]>-\epsilon\right\} \\
& =\left\{\sup _{t \in[\sigma, T]}\left[\theta^{+}\left(\bar{S}_{t}-\underline{S}_{\sigma}\right)-\theta^{-}\left(\underline{S}_{t}-\bar{S}_{\sigma}\right)\right]<\epsilon\right\} .
\end{aligned}
$$

This means that

$$
\mathbb{P}\left(\sup _{t \in[\sigma, T]}\left[\theta^{+}\left(\bar{S}_{t}-\underline{S}_{\sigma}\right)-\theta^{-}\left(\underline{S}_{t}-\bar{S}_{\sigma}\right)\right]<\epsilon \mid \mathcal{F}_{\sigma}\right)>0 \quad \mathbb{P} \text {-a.s. }
$$

The next definition introduces the notion of 0 -admissible strategy.

DeFinition 2.5. We say that a simple strategy $\Theta$ is 0 -admissible if

$$
\mathbb{P}\left(\inf _{t \in[0, T]} V_{t}(\Theta) \geq 0\right)=1 .
$$


Lemma 2.6. Let $\underline{S}$ and $\bar{S}$ be adapted càdlàg processes. If $(\underline{S}, \bar{S})$ satisfies condition $(\mathrm{C})$, then every simple arbitrage is 0-admissible.

This result is a bid and ask prices version of [2, Proposition 2.6].

Proof. Suppose $\Theta_{t}=\sum_{i=1}^{n-1} \theta_{i} \chi_{\left(\tau_{i}, \tau_{i+1}\right]}(t)$ is a simple arbitrage which is not 0 -admissible. Then there exists $i \in\{1, \ldots, n-1\}$ such that

$$
\mathbb{P}\left(\inf _{t \in\left[\tau_{i}, \tau_{i+1}\right)} V_{t}(\Theta)<0\right)>0 .
$$

Indeed, otherwise for all $i \in\{1, \ldots, n-1\}$ we have $\inf _{t \in\left[\tau_{i}, \tau_{i+1}\right)} V_{t}(\Theta) \geq 0$ $\mathbb{P}$-almost surely and $\mathbb{P}\left(\inf _{t \in[0, T]} V_{t}(\Theta) \geq 0\right)=1$, contradicting the non-0admissibility of $\Theta$. We define

$$
j_{0}=\max \left\{i=1, \ldots, n-1: \mathbb{P}\left(\inf _{t \in\left[\tau_{i}, \tau_{i+1}\right)} V_{t}(\Theta)<0\right)>0\right\} .
$$

Let $\tau_{j_{0}}:=\rho$ and $\tau_{j_{0+1}}:=\tau$. Observe that by the definition of $j_{0}$ we have $V_{\tau}(\Theta) \geq 0 \mathbb{P}$-almost surely. Furthermore there is a $\delta>0$ such that the set $\left\{\inf _{t \in[\rho, \tau)} V_{t}(\Theta) \leq-2 \delta\right\}$ has a positive probability. Define

$$
\sigma=\inf \left\{t>\rho: V_{t}(\Theta) \leq-\delta\right\} \wedge \tau .
$$

Note that $V_{t}(\Theta)$ is right continuous on $(\rho, \tau)$, so $V_{\sigma}(\Theta) \leq-\delta$ whenever $\sigma>\rho$ and since at a given time, immediate buying and selling is never optimal, we have $V_{\sigma}(\Theta) \leq V_{\sigma}^{+}(\Theta) \leq-\delta$ when $\sigma=\rho$, where $V_{\sigma}^{+}$denotes the right hand limit of $V_{t}$ at time $\sigma$. Therefore

$$
\delta \leq V_{\tau}(\Theta)-V_{\sigma}(\Theta)
$$

on $\{\sigma<\tau\} \in \mathcal{F}_{\sigma}$ (moreover $\mathbb{P}(\sigma<\tau)>0$ ). On the other hand,

$$
\begin{aligned}
& V_{\tau}(\Theta)=\sum_{i=1}^{j_{0}}\left[-\left(\theta_{i}-\theta_{i-1}\right)^{+} \bar{S}_{\tau_{i}}+\left(\theta_{i}-\theta_{i-1}\right)^{-} \underline{S}_{\tau_{i}}\right]+\theta_{j_{0}}^{+} \underline{S}_{\tau}-\theta_{j_{0}}^{-} \bar{S}_{\tau}, \\
& V_{\sigma}(\Theta)=\sum_{i=1}^{j_{0}}\left[-\left(\theta_{i}-\theta_{i-1}\right)^{+} \bar{S}_{\tau_{i}}+\left(\theta_{i}-\theta_{i-1}\right)^{-} \underline{S}_{\tau_{i}}\right]+\theta_{j_{0}}^{+} \underline{S}_{\sigma}-\theta_{j_{0}}^{-} \bar{S}_{\sigma},
\end{aligned}
$$

and on $\{\sigma<\tau\}$ we have

$$
\begin{aligned}
& V_{\tau}(\Theta)-V_{\sigma}(\Theta)=\theta_{j_{0}}^{+}\left(\underline{S}_{\tau}-\underline{S}_{\sigma}\right)+\theta_{j_{0}}^{-}\left(\bar{S}_{\sigma}-\bar{S}_{\tau}\right) \\
& \quad \leq \theta_{j_{0}}^{+}\left(\bar{S}_{\tau}-\underline{S}_{\sigma}\right)+\theta_{j_{0}}^{-}\left(\bar{S}_{\sigma}-\underline{S}_{\tau}\right)=\theta_{j_{0}}^{+}\left(\bar{S}_{\tau}-\underline{S}_{\sigma}\right)-\theta_{j_{0}}^{-}\left(\underline{S}_{\tau}-\bar{S}_{\sigma}\right) .
\end{aligned}
$$

We fix a sufficiently large $K$ such that $\mathbb{P}\left(\{\sigma<\tau\} \cap\left\{0<\left|\theta_{j_{0}}(\omega)\right| \leq K\right\}\right)>0$. Let $A:=\{\sigma<\tau\} \cap\left\{0<\left|\theta_{j_{0}}(\omega)\right| \leq K\right\}$. Clearly $A \in \mathcal{F}_{\sigma}$. Now we define

$$
\eta(\omega)= \begin{cases}\theta_{j_{0}}(\omega) /\left|\theta_{j_{0}}(\omega)\right|, & \omega \in A, \\ 1, & \omega \notin A .\end{cases}
$$


Then, on $A$,

$$
\begin{aligned}
\delta & \leq V_{\tau}(\Theta)-V_{\sigma}(\Theta) \leq \theta_{j_{0}}^{+}\left(\bar{S}_{\tau}-\underline{S}_{\sigma}\right)-\theta_{j_{0}}^{-}\left(\underline{S}_{\tau}-\bar{S}_{\sigma}\right) \\
& \leq K\left[\eta^{+}\left(\bar{S}_{\tau}-\underline{S}_{\sigma}\right)-\eta^{-}\left(\underline{S}_{\tau}-\bar{S}_{\sigma}\right)\right] .
\end{aligned}
$$

Consequently,

$$
\begin{aligned}
\mathbb{P}\left(A \cap\left\{\sup _{t \in[\sigma, T]} \eta^{+}\left(\bar{S}_{t}-\underline{S}_{\sigma}\right)-\eta^{-}\left(\underline{S}_{t}-\bar{S}_{\sigma}\right)<\delta / K\right\}\right) \\
\quad \leq \mathbb{P}\left(A \cap\left\{\eta^{+}\left(\bar{S}_{\tau}-\underline{S}_{\sigma}\right)-\eta^{-}\left(\underline{S}_{\tau}-\bar{S}_{\sigma}\right)<\delta / K\right\}\right)=0,
\end{aligned}
$$

which, by Remark 2.4, contradicts condition (C).

LEMmA 2.7. Let $\underline{S}$ and $\bar{S}$ be adapted càdlàg processes such that for every $t \in[0, T], \underline{S}_{t}<\bar{S}_{t} \mathbb{P}$-almost surely, which means that we have a strict inequality for each coordinate. Then no nontrivial simple strategy is 0-admissible.

Proof. Suppose $\Theta_{t}=\sum_{i=1}^{n-1} \theta_{i} \chi_{\left(\tau_{i}, \tau_{i+1}\right]}(t)$ is a 0 -admissible simple strategy such that $\mathbb{P}\left(\theta_{i} \neq 0\right)>0$ for some $i \in\{1, \ldots, n-1\}$. Let

$$
k_{0}=\min \left\{i=1, \ldots, n-1: \mathbb{P}\left(\theta_{i} \neq 0\right)>0\right\} .
$$

Then we have $V_{t}(\Theta)=0 \mathbb{P}$-almost surely for every $t \in\left[0, \tau_{k_{0}}\right]$, and $V_{t}(\Theta)=$ $\theta_{k_{0}}^{+}\left(\underline{S}_{t}-\bar{S}_{\tau_{k_{0}}}\right)-\theta_{k_{0}}^{-}\left(\bar{S}_{t}-\underline{S}_{\tau_{k_{0}}}\right)$ for $t \in\left(\tau_{k_{0}}, \tau_{k_{0}+1}\right]$. Since $\underline{S}$ and $\bar{S}$ are càdlàg processes, it follows that

$$
\lim _{t \rightarrow \tau_{k_{0}}^{+}} V_{t}(\Theta)=\theta_{k_{0}}^{+}\left(\underline{S}_{\tau_{k_{0}}}-\bar{S}_{\tau_{k_{0}}}\right)-\theta_{k_{0}}^{-}\left(\bar{S}_{\tau_{k_{0}}}-\underline{S}_{\tau_{k_{0}}}\right)=\left|\theta_{k_{0}}\right|\left(\underline{S}_{\tau_{k_{0}}}-\bar{S}_{\tau_{k_{0}}}\right) .
$$

Therefore $\lim _{t \rightarrow \tau_{k_{0}}^{+}} V_{t}(\Theta) \leq 0 \mathbb{P}$-almost surely. Let $A=\left\{\underline{S}_{\tau_{k_{0}}}<\bar{S}_{\tau_{k_{0}}}\right\} \cap$ $\left\{\theta_{k_{0}} \neq 0\right\}$. Then $\mathbb{P}(A)>0$ and $\lim _{t \rightarrow \tau_{k_{0}}^{+}} V_{t}(\Theta)<0$ on $A$. Hence

$$
0<\mathbb{P}(A)=\mathbb{P}\left(A \cap\left\{\inf _{t \in\left(\tau_{k_{0}}, \tau_{k_{0}+1}\right]} V_{t}(\Theta)<0\right\}\right) \leq \mathbb{P}\left(\inf _{t \in[0, T]} V_{t}(\Theta)<0\right),
$$

which contradicts the assumption

$$
\mathbb{P}\left(\inf _{t \in[0, T]} V_{t}(\Theta) \geq 0\right)=1 .
$$

Summarizing the last two lemmas we obtain

Corollary 2.8. Let $\underline{S}$ and $\bar{S}$ be adapted càdlàg processes such that for every $t \in[0, T], \underline{S}_{t}<\bar{S}_{t} \overline{\mathbb{P}}$-almost surely. If $(\underline{S}, \bar{S})$ satisfies condition $(\mathrm{C})$, then there is no simple arbitrage with bid and ask processes $\underline{S}$ and $\bar{S}$.

Proof. Assume $\underline{S}$ and $\bar{S}$ satisfy condition (C) and

$$
\Theta_{t}=\sum_{i=1}^{n-1} \theta_{i} \chi_{\left(\tau_{i}, \tau_{i+1}\right]}(t)
$$

is a simple arbitrage. Then, by Lemma 2.6, the strategy $\Theta$ is 0 -admissible. 
On the other hand, since $\mathbb{P}\left(\theta_{i} \neq 0\right)>0$ for some $i \in\{1, \ldots, n-1\}, \Theta$ is not 0 -admissible (by Lemma 2.7). This contradiction completes the proof.

3. Arbitrage for generalized almost simple strategies on the market with bid and ask prices. The following lemma shows that if some price process $S$ lies between bid and ask processes $(\underline{S}, \bar{S})$, then trading on $(\underline{S}, \bar{S})$ using a generalized almost simple strategy is no better than generalized almost simple trading on $S$ without transaction costs. A similar observation for simple trading strategies can be found in [4, Lemma 2.5].

Lemma 3.1. Let $S=\left(S_{t}\right)_{t \in[0, T]}$ be a process such that for any $t \in[0, T]$,

$$
\underline{S}_{t} \leq S_{t} \leq \bar{S}_{t} \quad \mathbb{P} \text {-a.s. }
$$

For any generalized almost simple investment strategy $\Theta=\left(\Theta_{t}\right)_{t \in[0, T]}$ of the form (1.4) and $s=\inf \left\{i \geq 1: \tau_{i}=1\right\}$ with $\theta_{s}=0$ we have

$$
V_{T}(\theta) \leq \sum_{i=2}^{s}\left[-\left(\theta_{i}^{-}-\theta_{i-1}\right) S_{\tau_{i}^{-}}\right]+\sum_{i=1}^{s}\left[-\left(\theta_{i}-\theta_{i}^{-}\right) S_{\tau_{i}}\right] .
$$

Moreover, if for any $t \in[0, T]$,

$$
\underline{S}_{t}<S_{t}<\bar{S}_{t} \quad \mathbb{P} \text {-a.s. }
$$

then

$$
V_{T}(\theta)<\sum_{i=2}^{s}\left[-\left(\theta_{i}^{-}-\theta_{i-1}\right) S_{\tau_{i}^{-}}\right]+\sum_{i=1}^{s}\left[-\left(\theta_{i}-\theta_{i}^{-}\right) S_{\tau_{i}}\right] \quad \mathbb{P} \text {-a.s. },
$$

with equality instead of strict inequality if and only if $\theta_{i}^{-}=\theta_{i}=0$ for all $i \geq 1$.

Proof. Observe that for $S=\left(S_{t}\right)_{t \in[0, T]}$ such that $\underline{S}_{t} \leq S_{t} \leq \bar{S}_{t}$ we have

$$
\begin{aligned}
\left(\theta_{i}^{-}-\theta_{i-1}\right)^{+} \bar{S}_{\tau_{i}^{-}} & \geq\left(\theta_{i}^{-}-\theta_{i-1}\right)^{+} S_{\tau_{i}^{-}} & & \text {for } i=2,3, \ldots, \\
\left(\theta_{i}-\theta_{i}^{-}\right)^{+} \bar{S}_{\tau_{i}} & \geq\left(\theta_{i}-\theta_{i}^{-}\right)^{+} S_{\tau_{i}} & & \text { for } i=1,2, \ldots, \\
\left(\theta_{i}^{-}-\theta_{i-1}\right)^{-} \underline{S}_{\tau_{i}^{-}} & \leq\left(\theta_{i}^{-}-\theta_{i-1}\right)^{-} S_{\tau_{i}^{-}} & & \text {for } i=2,3, \ldots, \\
\left(\theta_{i}-\theta_{i}^{-}\right)^{-} \underline{S}_{\tau_{i}} & \leq\left(\theta_{i}-\theta_{i}^{-}\right)^{-} S_{\tau_{i}} & & \text { for } i=1,2, \ldots,
\end{aligned}
$$

Then for $s=\min \left\{i \geq 1: \tau_{i}=T\right\}$ we have, using (1.6),

$$
\begin{aligned}
V_{T}(\Theta)= & \sum_{i=2}^{s}\left[-\left(\theta_{i}^{-}-\theta_{i-1}\right)^{+} \bar{S}_{\tau_{i}^{-}}+\left(\theta_{i}^{-}-\theta_{i-1}\right)^{-} \underline{S}_{\tau_{i}^{-}}\right] \\
& +\sum_{i=1}^{s}\left[-\left(\theta_{i}-\theta_{i}^{-}\right)^{+} \bar{S}_{\tau_{i}}+\left(\theta_{i}-\theta_{i}^{-}\right)^{-} \underline{S}_{\tau_{i}}\right] \\
\leq & \sum_{i=2}^{s}\left[-\left(\theta_{i}^{-}-\theta_{i-1}\right) S_{\tau_{i}^{-}}\right]+\sum_{i=1}^{s}\left[-\left(\theta_{i}-\theta_{i}^{-}\right) S_{\tau_{i}}\right] .
\end{aligned}
$$


We immediately deduce

Corollary 3.2. If there is a process $S$ such that $\underline{S}_{t} \leq S_{t} \leq \bar{S}_{t}$ for any $t \in[0, T]$ and there is no arbitrage at time $T$ for $S$ over generalized almost simple strategies then there is no arbitrage in the market with bid and ask prices $(\underline{S}, \bar{S})$.

The next corollary easily follows from the above in view of (1.7).

Corollary 3.3. If there exists a process $S=\left(S_{t}\right)_{t \in[0, T]}$ with

$$
\underline{S}_{t} \leq S_{t} \leq \bar{S}_{t} \quad \mathbb{P} \text {-a.s., } t \in[0, T],
$$

then for any almost simple investment strategy of the form

$$
\Theta_{t}=\sum_{i=1}^{\infty} \theta_{i} \chi_{\left(\tau_{i}, \tau_{i+1}\right]}(t)
$$

we have

$$
V_{T}(\Theta) \leq \sum_{i=1}^{s-1} \theta_{i}\left(S_{\tau_{i+1}}-S_{\tau_{i}}\right) \quad \text { where } \quad s=\inf \left\{i \geq 1: \tau_{i}=T\right\} .
$$

We also obtain

Corollary 3.4. If there is a price process $S$ such that $\underline{S}_{t} \leq S_{t} \leq \bar{S}_{t}$ for any $t \in[0, T]$ and there is an equivalent martingale measure for $S$ then there is no simple arbitrage in the market with bid and ask prices $(\underline{S}, \bar{S})$.

[13, Theorem 2.18] and Lemma 3.1 yield

Corollary 3.5. If there exists a càdlàg process $S$ such that

(i) $\underline{S}_{t} \leq S_{t} \leq \bar{S}_{t}$ for any $t \in[0, T]$,

(ii) for any stopping time $\tau$ with $\tau \leq T$ a.s. and any $\epsilon>0$ we have

$$
\mathbb{P}\left(\inf _{t \in[\tau, T]}\left(S_{t}-S_{\tau}\right)>-\epsilon \mid \mathcal{F}_{\tau}\right)>0 \quad \mathbb{P} \text {-a.s. }
$$

(iii) there is a probability measure $\mathbb{Q}$ equivalent to $\mathbb{P}$ such that $S$ is a local martingale with respect to $\mathbb{Q}$,

then there is no simple arbitrage in the market with bid and ask prices.

Definition 3.6. We say that a pair of adapted càdlàg processes $\underline{S}$ and $\bar{S}$ satisfies condition (D) with respect to the filtration $\mathbb{F}$ if for any stopping time $\tau$ such that $\tau \leq T$ a.s. there is an $\mathcal{F}_{\tau}$-measurable random variable $S_{\tau}$ such that

(i) $\mathbb{P}\left(\bigcap_{t \in[\tau, T]}\left\{S_{\tau} \in\left(\underline{S}_{t}, \bar{S}_{t}\right)\right\} \mid \mathcal{F}_{\tau}\right)>0 \mathbb{P}$-a.s.

(ii) $\underline{S}_{\tau^{-}} \leq S_{\tau} \leq \bar{S}_{\tau^{-}} \mathbb{P}$-a.s. 
Proposition 3.7. Under (D) there is no arbitrage at time $T$ with respect to generalized almost simple strategies in the market with bid and ask prices $(\underline{S}, \bar{S})$.

Proof. Assume $\Theta \in g a \mathcal{S}_{T}$ is an arbitrage strategy. Then $V_{T}(\Theta) \geq 0 \mathbb{P}$-a.s. and $\mathbb{P}\left(V_{T}(\Theta)>0\right)>0$. Let $\sigma=\min \left\{i \geq 1: \theta_{i}^{-} \neq 0 \vee \theta_{i} \neq 0\right\}$ and $s=$ $\min \left\{i \geq 1: \tau_{i}=T\right\}$. Since $\mathbb{P}\left(V_{T}(\Theta)>0\right)>0$ and $\left\{V_{T}(\Theta)>0\right\} \subset\left\{\tau_{\sigma} \leq \tau_{s}\right\}$, the event $A=\left\{\tau_{\sigma} \leq \tau_{s}\right\}$ has a positive probability. Observe that $A \in \mathcal{F}_{\tau_{\sigma}}$. We can write

$$
\begin{aligned}
V_{T}(\Theta)= & \sum_{i=\sigma}^{s}\left[-\left(\theta_{i}^{-}-\theta_{i-1}\right)^{+} \bar{S}_{\tau_{i}^{-}}+\left(\theta_{i}^{-}-\theta_{i-1}\right)^{-} \underline{S}_{\tau_{i}^{-}}\right] \\
& +\sum_{i=\sigma}^{s}\left[-\left(\theta_{i}-\theta_{i}^{-}\right)^{+} \bar{S}_{\tau_{i}}+\left(\theta_{i}-\theta_{i}^{-}\right)^{-} \underline{S}_{\tau_{i}}\right]
\end{aligned}
$$

where $\theta_{\sigma-1}=\theta_{s}=0$. Note that

$$
\sum_{i=\sigma}^{s}\left(\theta_{i}-\theta_{i-1}\right)=\sum_{i=\sigma}^{s}\left(\theta_{i}-\theta_{i}^{-}\right)+\sum_{i=\sigma}^{s}\left(\theta_{i}^{-}-\theta_{i-1}\right)=\theta_{s}-\theta_{\sigma-1}=0 .
$$

Hence

$$
\sum_{i=\sigma}^{s}\left(\theta_{i}-\theta_{i}^{-}\right)^{+}-\sum_{i=\sigma}^{s}\left(\theta_{i}-\theta_{i}^{-}\right)^{-}+\sum_{i=\sigma}^{s}\left(\theta_{i}^{-}-\theta_{i-1}\right)^{+}-\sum_{i=\sigma}^{s}\left(\theta_{i}^{-}-\theta_{i-1}\right)^{-}=0 .
$$

It follows that

$$
\begin{aligned}
V_{T}(\Theta)= & \sum_{i=\sigma}^{s}\left[-\left(\theta_{i}^{-}-\theta_{i-1}\right)^{+}\left(\bar{S}_{\tau_{i}^{-}}-S_{\tau_{\sigma}}\right)+\left(\theta_{i}^{-}-\theta_{i-1}\right)^{-}\left(\underline{S}_{\tau_{i}^{-}}-S_{\tau_{\sigma}}\right)\right] \\
& +\sum_{i=\sigma}^{s}\left[-\left(\theta_{i}-\theta_{i}^{-}\right)^{+}\left(\bar{S}_{\tau_{i}}-S_{\tau_{\sigma}}\right)+\left(\theta_{i}-\theta_{i}^{-}\right)^{-}\left(\underline{S}_{\tau_{i}}-S_{\tau_{\sigma}}\right)\right] .
\end{aligned}
$$

Let

$$
C=\bigcap_{t \in\left[\tau_{\sigma}, T\right]}\left\{S_{\tau_{\sigma}} \in\left(\underline{S}_{t}, \bar{S}_{t}\right)\right\} \cap\left\{S_{\tau_{\sigma}} \in\left[\underline{S}_{\tau_{\sigma}^{-}}, \bar{S}_{\tau_{\sigma}^{-}}\right]\right\} \cap A .
$$

Since the processes $\underline{S}$ and $\bar{S}$ satisfy condition (D), the event $C$ has positive probability. On $C$ we have

$$
\begin{aligned}
& \left(\theta_{i}^{-}-\theta_{i-1}\right)^{+} S_{\tau_{\sigma}} \leq\left(\theta_{i}^{-}-\theta_{i-1}\right)^{+} \bar{S}_{\tau_{i}^{-}}, \\
& \left(\theta_{i}^{-}-\theta_{i-1}\right)^{-} \underline{S}_{\tau_{i}^{-}} \leq\left(\theta_{i}^{-}-\theta_{i-1}\right)^{-} S_{\tau_{\sigma}}, \\
& \left(\theta_{i}-\theta_{i}^{-}\right)^{+} S_{\tau_{\sigma}} \leq\left(\theta_{i}-\theta_{i}^{-}\right)^{+} \bar{S}_{\tau_{i}}, \\
& \left(\theta_{i}-\theta_{i}^{-}\right)^{-} S_{\tau_{\sigma}} \leq\left(\theta_{i}-\theta_{i}^{-}\right)^{-} \bar{S}_{\tau_{i}},
\end{aligned}
$$

for $i=\sigma, \ldots, s$, with at least one strict inequality. Consequently, using (3.1) we find that $V_{T}(\Theta)<0$ on the set $C$, a contradiction. 
REMARK 3.8. A sufficient condition for the absence of arbitrage over almost simple trading strategies on the market with bid and ask prices $(\underline{S}, \bar{S})$ is the following: for any stopping time $\tau$ such that $\tau \leq T$ a.s. there is an $\mathcal{F}_{\tau}$-measurable random variable $S_{\tau}$ such that

$$
\mathbb{P}\left(\bigcap_{t \in[\tau, T]}\left\{S_{\tau} \in\left(\underline{S}_{t}, \bar{S}_{t}\right)\right\} \mid \mathcal{F}_{\tau}\right)>0 \quad \mathbb{P} \text {-a.s. }
$$

4. Arbitrage for simple strategies without shortselling. In this section we consider the case when our investment strategies are nonnegative, i.e. we are not allowed to borrow assets.

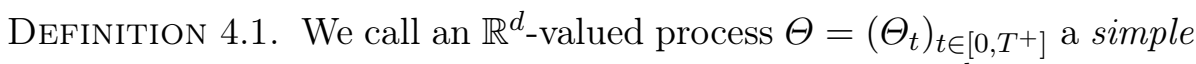
investment strategy without shortselling if $\Theta \in \mathcal{S}_{T}$ and $\theta_{i} \in \mathbb{R}^{d}$ are nonnegative $\mathcal{F}_{\tau_{i}}$-measurable random vectors.

The set of simple investment strategies without shortselling is denoted by $\mathcal{S}_{T}^{+}$.

Definition 4.2. We say that $(\underline{S}, \bar{S})$ admits an arbitrage opportunity with respect to the class of simple strategies without shortselling if there exists $\Theta \in \mathcal{S}_{T}^{+}$such that 1.9 holds.

The following is a slightly modified version of [13, Lemma 3.7].

Proposition 4.3. Let $\underline{\xi}_{1}, \bar{\xi}_{1}, \ldots, \underline{\xi}_{n}, \bar{\xi}_{n}$ be a sequence of nonnegative random variables on a filtered probability space $\left(\Omega, \mathcal{F}, \mathbb{P}, \mathbb{F}=\left(\mathcal{F}_{i}\right)_{i \in\{0, \ldots, n\}}\right)$. Let $\left(\eta_{i}\right)_{i=0}^{n}$ be a sequence of nonnegative random variables such that $\eta_{0}=\eta_{n}=0$ and $\eta_{i}$ are $\mathcal{F}_{i-1}$-measurable random variables and $\mathbb{P}\left(\eta_{i}>0\right)>0$ for some $i \in\{1, \ldots, n-1\}$. Then

$$
\bigcap_{\substack{i, j=1 \\ i<j}}^{n}\left\{\underline{\xi}_{j}<\bar{\xi}_{i}\right\} \subset\left\{V_{\underline{\xi}_{1}, \bar{\xi}_{1}, \ldots, \underline{\xi}_{n}, \bar{\xi}_{n}}\left(\eta_{0}, \eta_{1}, \ldots, \eta_{n}\right)<0\right\}
$$

where

$$
V_{\underline{\xi}_{1}, \bar{\xi}_{1}, \ldots, \underline{\xi}_{n}, \bar{\xi}_{n}}\left(\eta_{0}, \eta_{1}, \ldots, \eta_{n}\right)=\sum_{i=1}^{n}\left[-\left(\eta_{i}-\eta_{i-1}\right)^{+} \bar{\xi}_{i}+\left(\eta_{i}-\eta_{i-1}\right)^{-} \underline{\xi}_{i}\right] .
$$

Proof. We use induction on $n$. First we prove the statement for $n=2$. Since $\eta_{0}=\eta_{2}=0$ and $\eta_{1}>0$ a.s., we have

$$
V_{\underline{\xi}_{1}, \bar{\xi}_{1}, \underline{\xi}_{2}, \bar{\xi}_{2}}\left(\eta_{0}, \eta_{1}, \eta_{2}\right)=\eta_{1}\left[\underline{\xi}_{2}-\bar{\xi}_{1}\right]<0
$$

on the set $\left\{\underline{\xi}_{2}<\bar{\xi}_{1}\right\}$. Now suppose that the assertion holds true for $k-1$. 
We consider first the process $V_{\xi_{1}}, \bar{\xi}_{1}, \ldots, \underline{\xi}_{k}, \bar{\xi}_{k}\left(\eta_{0}, \eta_{1}, \ldots, \eta_{k}\right) \chi_{\left\{\eta_{1}=\eta_{2}\right\}}$. Clearly

$$
\begin{aligned}
V_{\xi_{1}}, \bar{\xi}_{1}, \ldots, \underline{\xi}_{k}, \bar{\xi}_{k}\left(\eta_{0}, \eta_{1}, \ldots,\right. & \left.\eta_{k}\right) \chi_{\left\{\eta_{1}=\eta_{2}\right\}} \\
& =V_{\underline{\xi}_{1}, \bar{\xi}_{1}, \underline{\xi}_{3}, \bar{\xi}_{3}, \ldots, \underline{\xi}_{k}, \bar{\xi}_{k}}\left(\eta_{0}, \eta_{1}, \eta_{3}, \ldots, \eta_{k}\right) \chi_{\left\{\eta_{1}=\eta_{2}\right\}}
\end{aligned}
$$

and

$$
\bigcap_{\substack{i, j=1 \\ i<j}}^{k}\left\{\underline{\xi}_{j}<\bar{\xi}_{i}\right\} \subset \bigcap_{\substack{i, j \in\{1, \ldots, n\} \backslash\{2\} \\ i<j}}\left\{\underline{\xi}_{j}<\bar{\xi}_{i}\right\},
$$

so by the induction hypothesis we have

$$
\begin{aligned}
\bigcap_{\substack{i, j=1 \\
i<j}}^{k}\left\{\underline{\xi}_{j}<\bar{\xi}_{i}\right\} \cap\left\{\eta_{1}\right. & \left.=\eta_{2}\right\} \\
& \subset\left\{V_{\underline{\xi}_{1}, \bar{\xi}_{1}, \ldots, \underline{\xi}_{k}, \bar{\xi}_{k}}\left(\eta_{0}, \eta_{1}, \ldots, \eta_{k}\right) \chi_{\left\{\eta_{1}=\eta_{2}\right\}}<0\right\} .
\end{aligned}
$$

The next step consists in considering the process

$$
\begin{aligned}
& V_{\xi_{1}, \bar{\xi}_{1}, \ldots, \underline{\xi}_{k}, \bar{\xi}_{k}}\left(\eta_{0}, \eta_{1}, \ldots, \eta_{k}\right) \chi_{\left\{\eta_{2}<\eta_{1}\right\}} \\
& =\chi_{\left\{\eta_{2}<\eta_{1}\right\}} \sum_{i=1}^{k}\left[-\left(\eta_{i}-\eta_{i-1}\right)^{+} \bar{\xi}_{i}+\left(\eta_{i}-\eta_{i-1}\right)^{-} \underline{\xi}_{i}\right] \\
& =\chi_{\left\{\eta_{2}<\eta_{1}\right\}}\left\{-\eta_{1} \bar{\xi}_{1}-\left(\eta_{2}-\eta_{1}\right) \underline{\xi}_{2}+\sum_{i=3}^{k}\left[-\left(\eta_{i}-\eta_{i-1}\right)^{+} \bar{\xi}_{i}+\left(\eta_{i}-\eta_{i-1}\right)^{-} \underline{\xi}_{i}\right]\right\}
\end{aligned}
$$

Note that

$$
\eta_{1}\left[\underline{\xi}_{2}-\bar{\xi}_{1}\right]<\eta_{2}\left[\underline{\xi}_{2}-\bar{\xi}_{1}\right]
$$

on the set $\left\{\underline{\xi}_{2}<\bar{\xi}_{1}\right\} \cap\left\{\eta_{2}<\eta_{1}\right\}$, from which it follows that

$$
\begin{aligned}
& V_{\underline{\xi}_{1}, \bar{\xi}_{1}, \ldots, \underline{\xi}_{k}, \bar{\xi}_{k}}\left(\eta_{0}, \eta_{1}, \ldots, \eta_{k}\right) \chi_{\left\{\eta_{2}<\eta_{1}\right\}} \\
& <\chi_{\left\{\eta_{2}<\eta_{1}\right\}}\left\{-\eta_{2} \bar{\xi}_{1}+\sum_{i=3}^{k}\left[-\left(\eta_{i}-\eta_{i-1}\right)^{+} \bar{\xi}_{i}+\left(\eta_{i}-\eta_{i-1}\right)^{-} \underline{\xi}_{i}\right]\right\} .
\end{aligned}
$$

Observe that the right hand side represents $V_{\underline{\xi}_{1}}, \bar{\xi}_{1}, \underline{\xi}_{3}, \bar{\xi}_{3}, \ldots, \underline{\xi}_{k}, \bar{\xi}_{k}\left(\eta_{0}, \eta_{2}, \ldots, \eta_{k}\right)$ on the set $\left\{\eta_{2}<\eta_{1}\right\}$, and hence

$$
\begin{aligned}
& \bigcap_{\substack{i, j=1 \\
i<j}}^{k}\left\{\underline{\xi}_{j}<\bar{\xi}_{i}\right\} \cap\left\{\eta_{2}<\eta_{1}\right\} \subset\left\{V_{\underline{\xi}_{1}}, \bar{\xi}_{1}, \ldots, \underline{\xi}_{k}, \bar{\xi}_{k}\left(\eta_{0}, \eta_{1}, \ldots, \eta_{k}\right) \chi_{\left\{\eta_{2}<\eta_{1}\right\}}\right. \\
& \left.<V_{\underline{\xi}_{1}}, \bar{\xi}_{1}, \underline{\xi}_{3}, \bar{\xi}_{3}, \ldots, \underline{\xi}_{k}, \bar{\xi}_{k}\left(\eta_{0}, \eta_{2}, \ldots, \eta_{k}\right) \chi_{\left\{\eta_{2}<\eta_{1}\right\}}\right\}
\end{aligned}
$$


By the induction hypothesis we have

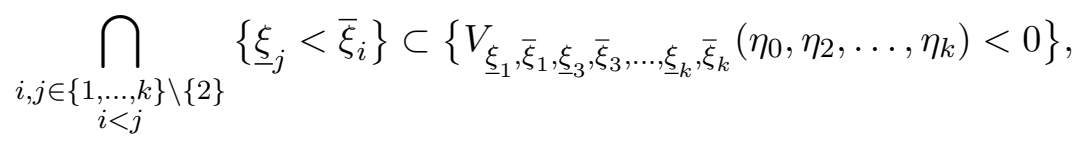

which means that

$$
\begin{aligned}
\bigcap_{\substack{i, j=1 \\
i<j}}^{k}\left\{\underline{\xi}_{j}<\bar{\xi}_{i}\right\} \cap\left\{\eta_{2}<\eta_{1}\right\} & \\
& \subset\left\{V_{\underline{\xi}_{1}, \bar{\xi}_{1}, \ldots, \underline{\xi}_{k}, \bar{\xi}_{k}}\left(\eta_{0}, \eta_{1}, \ldots, \eta_{k}\right) \chi_{\left\{\eta_{2}<\eta_{1}\right\}}<0\right\} .
\end{aligned}
$$

Now we consider the process $V_{\xi_{1}, \bar{\xi}_{1}, \ldots, \underline{\xi}_{k}, \bar{\xi}_{k}}\left(\eta_{0}, \eta_{1}, \ldots, \eta_{k}\right) \chi_{\left\{\eta_{2}>\eta_{1}\right\}}$. Let

$$
l=\max \left\{s: \eta_{1}<\eta_{2} \leq \cdots \leq \eta_{s-1}\right\} .
$$

Note that $l$ is well defined because we have assumed that $\eta_{0}, \eta_{1}, \ldots, \eta_{k}$ are nonnegative random variables and $\eta_{k}=0$. Clearly,

$$
\begin{aligned}
& V_{\underline{\xi}_{1}, \bar{\xi}_{1}, \ldots, \underline{\xi}_{k}, \bar{\xi}_{k}}\left(\eta_{0}, \eta_{1}, \ldots, \eta_{k}\right) \chi_{\left\{\eta_{2}>\eta_{1}\right\}} \\
& =\chi_{\left\{\eta_{2}>\eta_{1}\right\}}\left\{\sum_{i=1}^{\tilde{l}-2}\left[-\left(\eta_{i}-\eta_{i-1}\right) \bar{\xi}_{i}\right]-\left(\eta_{\tilde{l}-1}-\eta_{\tilde{l}-2}\right) \bar{\xi}_{\tilde{l}-1}-\left(\eta_{\tilde{l}}-\eta_{\tilde{l}-1}\right) \underline{\xi}_{\tilde{l}}\right. \\
& \left.\quad+\sum_{i=\tilde{l}+1}^{k}\left[-\left(\eta_{i}-\eta_{i-1}\right)^{+} \bar{\xi}_{i}+\left(\eta_{i}-\eta_{i-1}\right)^{-} \underline{\xi}_{i}\right]\right\}
\end{aligned}
$$

on the set $\{l=\tilde{l}\}$ for some $\tilde{l} \in\{3, \ldots, k\}$. Observe that

$$
\begin{aligned}
-\left(\eta_{\tilde{l}-1}-\eta_{\tilde{l}-2}\right) \bar{\xi}_{\tilde{l}-1}-\left(\eta_{\tilde{l}}-\eta_{\tilde{l}-1}\right) \underline{\xi}_{\tilde{l}} & \\
& =-\left(\eta_{\tilde{l}-1}-\eta_{\tilde{l}}\right) \bar{\xi}_{\tilde{l}-1}-\left(\eta_{\tilde{l}}-\eta_{\tilde{l}-2}\right) \bar{\xi}_{\tilde{l}-1}-\left(\eta_{\tilde{l}}-\eta_{\tilde{l}-1}\right) \underline{\xi}_{\tilde{l}} \\
& =\left(\eta_{\tilde{l}-1}-\eta_{\tilde{l}}\right)\left(\underline{\xi}_{\tilde{l}}-\bar{\xi}_{\tilde{l}-1}\right)-\left(\eta_{\tilde{l}}-\eta_{\tilde{l}-2}\right) \bar{\xi}_{\tilde{l}-1} .
\end{aligned}
$$

Since

$$
\left(\eta_{\tilde{l}-1}-\eta_{\tilde{l}}\right)\left(\underline{\xi}_{\tilde{l}}-\bar{\xi}_{\tilde{l}-1}\right)<0
$$

on the set $\{l=\tilde{l}\} \cap\left\{\underline{\xi}_{\tilde{l}}<\bar{\xi}_{\tilde{l}-1}\right\}$, we have

$$
-\left(\eta_{\tilde{l}-1}-\eta_{\tilde{l}-2}\right) \bar{\xi}_{\tilde{l}-1}-\left(\eta_{\tilde{l}}-\eta_{\tilde{l}-1}\right) \underline{\xi}_{\tilde{l}}<-\left(\eta_{\tilde{l}}-\eta_{\tilde{l}-2}\right) \bar{\xi}_{\tilde{l}-1} .
$$

Similarly

and since

$$
\begin{aligned}
-\left(\eta_{\tilde{l}-1}-\eta_{\tilde{l}-2}\right) \bar{\xi}_{\tilde{l}-1}-\left(\eta_{\tilde{l}}-\eta_{\tilde{l}-1}\right) \underline{\xi}_{\tilde{l}} \\
\quad=-\left(\eta_{\tilde{l}-1}-\eta_{\tilde{l}-2}\right) \bar{\xi}_{\tilde{l}-1}-\left(\eta_{\tilde{l}}-\eta_{\tilde{l}-2}\right) \underline{\xi}_{\tilde{l}}-\left(\eta_{\tilde{l}-2}-\eta_{\tilde{l}-1}\right) \underline{\xi}_{\tilde{l}} \\
\quad=\left(\eta_{\tilde{l}-1}-\eta_{\tilde{l}-2}\right)\left(\underline{\xi}_{\tilde{l}}-\bar{\xi}_{\tilde{l}-1}\right)-\left(\eta_{\tilde{l}}-\eta_{\tilde{l}-2}\right) \underline{\xi}_{\tilde{l}},
\end{aligned}
$$

$$
\left(\eta_{\tilde{l}-1}-\eta_{\tilde{l}-2}\right)\left(\underline{\xi}_{\tilde{l}}-\bar{\xi}_{\tilde{l}-1}\right)<0
$$


on the set $\{l=\tilde{l}\} \cap\left\{\underline{\xi}_{\tilde{l}}<\bar{\xi}_{\tilde{l}-1}\right\} \cap\left\{\eta_{\tilde{l}-2}<\eta_{\tilde{l}-1}\right\}$, we have

$$
-\left(\eta_{\tilde{l}-1}-\eta_{\tilde{l}-2}\right) \bar{\xi}_{\tilde{l}-1}-\left(\eta_{\tilde{l}}-\eta_{\tilde{l}-1}\right) \underline{\xi}_{\tilde{l}}<-\left(\eta_{\tilde{l}}-\eta_{\tilde{l}-2}\right) \underline{\xi}_{\tilde{l}} \text {. }
$$

Now, using 4.3 we obtain, on the event $\{l=\tilde{l}\} \cap\left\{\underline{\xi}_{\tilde{l}}<\bar{\xi}_{\tilde{l}-1}\right\}$,

$V_{\underline{\xi}_{1}, \bar{\xi}_{1}, \ldots, \underline{\xi}_{k}, \bar{\xi}_{k}}\left(\eta_{0}, \eta_{1}, \ldots, \eta_{k}\right) \chi_{\left\{\eta_{2}>\eta_{1}\right\}} \chi_{\{l=\tilde{l}\}} \chi_{\left\{\eta_{l} \geq \eta_{l-2}\right\}}$

$<\chi_{\left\{\eta_{2}>\eta_{1}\right\}} \chi_{\{l=\tilde{l}\}} \chi_{\left\{\eta_{l} \geq \eta_{l-2}\right\}}\left\{\sum_{i=1}^{\tilde{l}-2}\left[-\left(\eta_{i}-\eta_{i-1}\right) \bar{\xi}_{i}\right]\right.$

$$
\left.-\left(\eta_{\tilde{l}}-\eta_{\tilde{l}-2}\right) \bar{\xi}_{\tilde{l}-1}+\sum_{i=\tilde{l}+1}^{k}\left[-\left(\eta_{i}-\eta_{i-1}\right)^{+} \bar{\xi}_{i}+\left(\eta_{i}-\eta_{i-1}\right)^{-} \underline{\xi}_{i}\right]\right\}
$$

$=V_{\underline{\xi}_{1}}, \bar{\xi}_{1}, \ldots, \underline{\xi}_{l-1}, \bar{\xi}_{l-1}, \underline{\xi}_{l+1} \bar{\xi}_{l+1}, \ldots, \underline{\xi}_{k}, \bar{\xi}_{k}\left(\eta_{0}, \ldots, \eta_{l-2}, \eta_{l}, \ldots, \eta_{k}\right) \chi_{\left\{\eta_{2}>\eta_{1}\right\}}$

$$
\times \chi_{\{l=\tilde{l}\}} \chi_{\left\{\eta_{l} \geq \eta_{l-2}\right\}},
$$

and by 4.4) we have

$$
\begin{aligned}
& V_{\xi_{1}}, \bar{\xi}_{1}, \ldots, \underline{\xi}_{k}, \bar{\xi}_{k}\left(\eta_{0}, \eta_{1}, \ldots, \eta_{k}\right) \chi_{\left\{\eta_{2}>\eta_{1}\right\}} \chi_{\{l=\tilde{l}\}} \chi_{\left\{\eta_{l}<\eta_{l-2}\right\}} \chi_{\left\{\eta_{l-2}<\eta_{l-1}\right\}} \\
& <\chi_{\left\{\eta_{2}>\eta_{1}\right\}} \chi_{\{l=\tilde{l}\}} \chi_{\left\{\eta_{l}<\eta_{l-2}\right\}} \chi_{\left\{\eta_{l-2}<\eta_{l-1}\right\}}\left\{\sum_{i=1}^{\tilde{l}-2}\left[-\left(\eta_{i}-\eta_{i-1}\right) \bar{\xi}_{i}\right]-\left(\eta_{\tilde{l}}-\eta_{\tilde{l}-2}\right) \underline{\xi}_{\tilde{l}}\right. \\
& \left.+\sum_{i=\tilde{l}+1}^{k}\left[-\left(\eta_{i}-\eta_{i-1}\right)^{+} \bar{\xi}_{i}+\left(\eta_{i}-\eta_{i-1}\right)^{-} \underline{\xi}_{i}\right]\right\} \\
& =V_{\underline{\xi}_{1}}, \bar{\xi}_{1}, \ldots, \underline{\xi}_{l-2}, \bar{\xi}_{l-2}, \underline{\xi}_{l}, \bar{\xi}_{l}, \ldots, \underline{\xi}_{k}, \bar{\xi}_{k}\left(\eta_{0}, \ldots, \eta_{l-2}, \eta_{l}, \ldots, \eta_{k}\right) \chi_{\left\{\eta_{2}>\eta_{1}\right\}} \chi_{\{l=\tilde{l}\}} \chi_{\left\{\eta_{l}<\eta_{l-2}\right\}} \\
& \times \chi\left\{\eta_{l-2}<\eta_{l-1}\right\}
\end{aligned}
$$

on the set $\{l=\tilde{l}\} \cap\left\{\underline{\xi}_{\tilde{l}}<\bar{\xi}_{\tilde{l}-1}\right\}$. Consider the case $\eta_{l}<\eta_{l-2}$ and $\eta_{l-1} \leq \eta_{l-2}$. Then (by the definition of $l$ ) $\eta_{l}<\eta_{l-1}=\eta_{l-2}$ and

$$
\begin{aligned}
& V_{\underline{\xi}_{1}, \bar{\xi}_{1}, \ldots, \underline{\xi}_{k}, \bar{\xi}_{k}}\left(\eta_{0}, \eta_{1}, \ldots, \eta_{k}\right) \chi_{\left\{\eta_{2}>\eta_{1}\right\}} \chi_{\{l=\tilde{l}\}} \chi_{\left\{\eta_{l}<\eta_{l-2}\right\}} \chi_{\left\{\eta_{l-2} \geq \eta_{l-1}\right\}} \\
& =\chi_{\left\{\eta_{2}>\eta_{1}\right\}} \chi_{\{l=\tilde{l}\}} \chi_{\left\{\eta_{l}<\eta_{l-2}\right\}} \chi_{\left\{\eta_{l-2} \geq \eta_{l-1}\right\}}\left\{\sum_{i=1}^{\tilde{l}-2}\left[-\left(\eta_{i}-\eta_{i-1}\right) \bar{\xi}_{i}\right]-\left(\eta_{\tilde{l}}-\eta_{\tilde{l}-2}\right) \underline{\xi}_{\tilde{l}}\right. \\
& \left.+\sum_{i=\tilde{l}+1}^{k}\left[-\left(\eta_{i}-\eta_{i-1}\right)^{+} \bar{\xi}_{i}+\left(\eta_{i}-\eta_{i-1}\right)^{-} \underline{\xi}_{i}\right]\right\} \\
& =V_{\underline{\xi}_{1}}, \bar{\xi}_{1}, \ldots, \underline{\xi}_{l-2}, \bar{\xi}_{l-2}, \underline{\xi}_{l}, \bar{\xi}_{l}, \ldots, \underline{\xi}_{k}, \bar{\xi}_{k}\left(\eta_{0}, \ldots, \eta_{l-2}, \eta_{l}, \ldots, \eta_{k}\right) \chi_{\left\{\eta_{2}>\eta_{1}\right\}} \chi_{\{l=\tilde{l}\}} \chi_{\left\{\eta_{l}<\eta_{l-2}\right\}} \\
& \times \chi\left\{\eta_{l-2} \geq \eta_{l-1}\right\} \text {. }
\end{aligned}
$$


The induction hypothesis then shows that

$$
\begin{aligned}
& \bigcap_{\substack{i, j=1 \\
i<j}}^{k}\left\{\underline{\xi}_{j}<\bar{\xi}_{i}\right\} \cap\left\{\eta_{2}>\eta_{1}\right\} \cap\{l=\tilde{l}\} \cap\left\{\eta_{l} \geq \eta_{l-2}\right\} \\
& \subset\left\{V_{\underline{\xi}_{1}, \bar{\xi}_{1}, \ldots, \underline{\xi}_{k}, \bar{\xi}_{k}}\left(\eta_{0}, \eta_{1}, \ldots, \eta_{k}\right) \chi_{\left\{\eta_{2}>\eta_{1}\right\}} \chi_{\{l=\tilde{l}\}} \chi_{\left\{\eta_{l} \geq \eta_{l-2}\right\}}<0\right\}, \\
& \bigcap_{\substack{i, j=1 \\
i<j}}^{k}\left\{\underline{\xi}_{j}<\bar{\xi}_{i}\right\} \cap\left\{\eta_{2}>\eta_{1}\right\} \cap\{l=\tilde{l}\} \cap\left\{\eta_{l}<\eta_{l-2}\right\} \cap\left\{\eta_{l-2}<\eta_{l-1}\right\} \\
& \quad \subset\left\{V_{\underline{\xi}_{1}, \bar{\xi}_{1}, \ldots, \underline{\xi}_{k}, \bar{\xi}_{k}}\left(\eta_{0}, \eta_{1}, \ldots, \eta_{k}\right) \chi_{\left\{\eta_{2}>\eta_{1}\right\}} \chi_{\{l=\tilde{l}\}} \chi_{\left\{\eta_{l}<\eta_{l-2}\right\}} \chi_{\left\{\eta_{l-2}<\eta_{l-1}\right\}}<0\right\} \\
& \text { and } \bigcap_{\substack{i, j=1 \\
i<j}}^{k}\left\{\underline{\xi}_{j}<\bar{\xi}_{i}\right\} \cap\left\{\eta_{2}>\eta_{1}\right\} \cap\{l=\tilde{l}\} \cap\left\{\eta_{l}<\eta_{l-2}\right\} \cap\left\{\eta_{l-2} \geq \eta_{l-1}\right\} \\
& \quad \subset\left\{V_{V_{\underline{\xi}_{1}}, \bar{\xi}_{1}, \ldots, \underline{\xi}_{k}, \bar{\xi}_{k}}\left(\eta_{0}, \eta_{1}, \ldots, \eta_{k}\right) \chi_{\left\{\eta_{2}>\eta_{1}\right\}} \chi_{\{l=\tilde{l}\}} \chi_{\left\{\eta_{l}<\eta_{l-2}\right\}} \chi_{\left\{\eta_{l-2} \geq \eta_{l-1}\right\}}<0\right\} .
\end{aligned}
$$

Hence,

$$
\begin{aligned}
\bigcap_{\substack{i, j=1 \\
i<j}}^{k}\left\{\underline{\xi}_{j}<\bar{\xi}_{i}\right\} & \cap\left\{\eta_{2}>\eta_{1}\right\} \cap\{l=\tilde{l}\} \\
& \subset\left\{V_{\underline{\xi}_{1}, \bar{\xi}_{1}, \ldots, \underline{\xi}_{k}, \bar{\xi}_{k}}\left(\eta_{0}, \eta_{1}, \ldots, \eta_{k}\right) \chi_{\left\{\eta_{2}>\eta_{1}\right\}} \chi_{\{l=\tilde{l}\}}<0\right\} .
\end{aligned}
$$

Combining 4.1), 4.2, 4.5) we obtain

$$
\bigcap_{\substack{i, j=1 \\ i<j}}^{k}\left\{\underline{\xi}_{j}<\bar{\xi}_{i}\right\} \subset\left\{V_{\underline{\xi}_{1},}, \bar{\xi}_{1}, \ldots, \underline{\xi}_{k}, \bar{\xi}_{k}\left(\eta_{0}, \eta_{1}, \ldots, \eta_{k}\right)<0\right\},
$$

which completes the proof.

We now impose another assumption:

Definition 4.4. We say that $(\underline{S}, \bar{S})$ satisfies condition $(\mathrm{E})$ with respect to the filtration $\mathbb{F}$ if for any stopping times $0=\tau_{1} \leq \cdots \leq \tau_{n}=T$ we have

$$
\mathbb{P}\left(\bigcap_{k=1}^{n}\left\{\underline{S}_{\tau_{k}}<\min _{j<k} \bar{S}_{\tau_{j}}\right\}\right)>0 .
$$

Lemma 4.5. If $(\underline{S}, \bar{S})$ satisfies condition $(\mathrm{E})$, then there is no simple arbitrage opportunity without shortselling on the market with bid and ask prices $(\underline{S}, \bar{S})$. 
Proof. Observe that

$$
\bigcap_{k=1}^{n}\left\{\underline{S}_{\tau_{k}}<\min _{j<k} \bar{S}_{\tau_{j}}\right\} \subset \bigcap_{\substack{j, k=1 \\ j<k}}^{k}\left\{\underline{S}_{\tau_{k}}<\bar{S}_{\tau_{j}}\right\} .
$$

Then we apply Proposition 4.3 to the sequence of random variables (playing the role of $\left(\underline{\xi}_{i}\right)$ and $\left.\left(\bar{\xi}_{i}\right)\right)$

$$
\underline{S}_{\tau_{1}}, \bar{S}_{\tau_{1}}, \ldots, \underline{S}_{\tau_{n}}, \bar{S}_{\tau_{n}}
$$

and the sequence of nonnegative random variables (standing for $\left(\eta_{i}\right)$ )

$$
\theta_{0}, \theta_{1}, \ldots, \theta_{n}
$$

where $\theta_{0}=\theta_{n}=0$.

We now introduce a sufficient condition for (E) to hold.

Definition 4.6. We say that $(\underline{S}, \bar{S})$ satisfies condition $(\mathrm{F})$ with respect to the filtration $\mathbb{F}$ if for any stopping times $\sigma<\tau \leq T$ and for any $x>0$ we have

$$
\mathbb{P}\left(\underline{S}_{\tau}<x \mid \mathcal{F}_{\sigma}\right)>0 \quad \mathbb{P} \text {-a.s. }
$$

Lemma 4.7. If $(\underline{S}, \bar{S})$ satisfies condition $(\mathrm{F})$, then there is no simple arbitrage without shortselling with bid and ask prices $(\underline{S}, \bar{S})$.

Proof. It follows from Lemma 4.5 that it suffices to show that if $(\underline{S}, \bar{S})$ satisfies condition (F) then $\mathbb{P}\left(\bigcap_{k=1}^{n}\left\{\underline{S}_{\tau_{k}}<\min _{j<k} \bar{S}_{\tau_{j}}\right\}\right)>0 \mathbb{P}$-a.s. for any stopping times $0=\tau_{1} \leq \cdots \leq \tau_{n}=T$. Suppose towards a contradiction that condition $(\mathrm{F})$ is satisfied and there exist stopping times $0=\tau_{1} \leq \cdots \leq \tau_{n}=T$ such that

$$
\mathbb{P}\left(\bigcap_{k=1}^{n}\left\{\underline{S}_{\tau_{k}}<\min _{j<k} \bar{S}_{\tau_{j}}\right\}\right)=0 .
$$

We show that for any $n>2$,

$$
\mathbb{P}\left(A_{n}\right)=0 \Rightarrow \mathbb{P}\left(A_{n-1}\right)=0,
$$

where $A_{n}=\bigcap_{k=1}^{n}\left\{\underline{S}_{\tau_{k}}<\min _{j<k} \bar{S}_{\tau_{j}}\right\} \in \mathcal{F}_{\tau_{n}}$. Observe that if

$$
0=\mathbb{P}\left(A_{n}\right)=\mathbb{P}\left(A_{n-1} \cap\left\{\underline{S}_{\tau_{n}}<\min _{j<n} \bar{S}_{\tau_{j}}\right\}\right)
$$

then

$$
\begin{aligned}
0 & =\mathbb{P}\left(\left\{\tau_{n}=\tau_{n-1}\right\} \cap A_{n-1} \cap\left\{\underline{S}_{\tau_{n}}<\min _{j<n} \bar{S}_{\tau_{j}}\right\}\right) \\
& =\mathbb{P}\left(\left\{\tau_{n}=\tau_{n-1}\right\} \cap A_{n-1}\right)
\end{aligned}
$$


and

$$
\begin{aligned}
0 & =E\left(\chi_{\left\{\tau_{n-1}<\tau_{n}\right\}} \chi_{A_{n-1}} \chi_{\left\{\underline{S}_{\tau_{n}}<\min _{j<n} \bar{S}_{\left.\tau_{j}\right\}} \mid \mathcal{F}_{\tau_{n-1}}\right)}\right. \\
& =\chi_{\left\{\tau_{n-1}<\tau_{n}\right\}} \chi_{A_{n-1}} \mathbb{P}\left(\underline{S}_{\tau_{n}}<\min _{j<n} \bar{S}_{\tau_{j}} \mid \mathcal{F}_{\tau_{n-1}}\right) .
\end{aligned}
$$

Since $\mathbb{P}\left(\underline{S}_{\tau_{n}}<\min _{j<n} \bar{S}_{\tau_{j}} \mid \mathcal{F}_{\tau_{n-1}}\right)>0$ provided that $\left\{\tau_{n-1}<\tau_{n}\right\}$, we should have

$$
\mathbb{P}\left(\left\{\tau_{n-1}<\tau_{n}\right\} \cap A_{n-1}\right)=0 .
$$

This together with 4.10 implies that $\mathbb{P}\left(A_{n-1}\right)=0$. From 4.8 and 4.9 it follows that $\mathbb{P}\left(A_{2}\right)=0$. Consider the two cases: (i) $\tau_{1}<\tau_{2} \mathbb{P}$-a.s. and (ii) $\mathbb{P}\left(\tau_{1}=\tau_{2}\right)>0$. Then in case (i),

$$
\mathbb{P}\left(\underline{S}_{\tau_{2}}<\bar{S}_{\tau_{1}}\right)=0
$$

contradicts condition (F), and in case (ii),

$$
0=\mathbb{P}\left(\left\{\tau_{1}=\tau_{2}\right\} \cap\left\{\underline{S}_{\tau_{2}}<\bar{S}_{\tau_{1}}\right\}\right)=\mathbb{P}\left(\left\{\underline{S}_{\tau_{1}}<\bar{S}_{\tau_{1}}\right\}\right)
$$

contradicts the assumption that $\underline{S}_{\tau_{1}}<\bar{S}_{\tau_{1}}$.

Acknowledgements. The research of A. Rygiel was financed from the funds granted to the Faculty of Finance and Law at Cracow University of Economics, within the framework of the subsidy for the maintenance of research potential. The research of $€$. Stettner was supported by NCN grant DEC-2012/07/B/ST1/03298.

\section{References}

[1] E. Bayraktar and H. Sayit, No arbitrage conditions for simple trading strategies, Ann. Finance 6 (2010), 147-156.

[2] C. Bender, Simple arbitrage, Ann. Appl. Probab. 22 (2012), 2067-2085.

[3] P. Cheredito, Arbitrage in fractional Brownian motion models, Finance Stoch. 7 (2003), 533-553.

[4] P. Guasoni, No arbitrage with transaction costs, with fractional Brownian motion and beyond, Math. Finance 16 (2006), 569-582.

[5] P. Guasoni, E. Lépinette and M. Rásonyi, The fundamental theorem of asset pricing under transaction costs, Finance Stoch. 16 (2012), 741-777.

[6] P. Guasoni, M. Rásonyi and W. Schachermayer, Consistent price systems and facelifting pricing under transaction costs, Ann. Appl. Probab. 18 (2008), 491-520.

[7] P. Guasoni, M. Rásonyi and W. Schachermayer, The fundamental theorem of asset pricing for continuous processes under small transaction costs, Ann. Finance 6 (2010), 157-191.

[8] S. He, J. Wang and J. Yan, Semimartingale Theory and Stochastic Calculus, CRC Press, 1992.

[9] J. Jacod and A. N. Shiryaev, Limit Theorems for Stochastic Processes, Springer, 2003. 
[10] R. Jarrow, P. Protter and H. Sayit, No arbitrage without semimartingales, Ann. Appl. Probab. 19 (2009), 596-616.

[11] C. Kardaras and E. Platen, On the semimartingale property of discounted asset-price processes, Stoch. Process. Appl. 121 (2011), 2678-2691.

[12] P. Rola, Arbitrage in markets with bid-ask spreads, Ann. Finance 11 (2015), 453-475.

[13] A. Rygiel and Ł. Stettner, Arbitrage for simple strategies, Appl. Math. (Warsaw) 39 (2012), 379-412.

[14] W. Schachermayer, Introduction to the mathematics of financial markets, in: Lectures on Probability Theory and Statistics (Saint-Flour, 2000), Lecture Notes in Math. 1816, Springer, Berlin, 2003, 107-179.

Agnieszka Rygiel

Department of Mathematics

Cracow University of Economics

31-510 Kraków, Poland

E-mail: Agnieszka.Rygiel@uek.krakow.pl
Łukasz Stettner Institute of Mathematics Polish Academy of Sciences 00-656 Warszawa, Poland and Vistula University E-mail: L.Stettner@impan.pl 
